\title{
A VARIANT OF D'ALEMBERT FUNCTIONAL EQUATION ON MONOIDS
}

\author{
CHAHBI ABDELLATIF AND ELQORACHI ELHOUCIEN
}

\begin{abstract}
In this paper, we determine the complex-valued solutions of the functional equation

$$
f(x \sigma(y))+f(\tau(y) x)=2 f(x) f(y)
$$

for all $x, y \in M$, where $M$ is a monoid, $\sigma: M \longrightarrow M$ is an involutive automorphism and $\tau: M \longrightarrow M$ is an involutive anti-automorphism. The solutions are expressed in terms of multiplicative functions, and characters of 2-dimensional irreducible representations of $M$.
\end{abstract}

\section{INTRODUCTION}

It is well-known that d'Alembert's functional equation

$$
f(x+y)+f(x-y)=2 f(x) f(y)
$$

for all $x, y \in \mathbb{R}$ has its continuous solutions $f: \mathbb{R} \longrightarrow \mathbb{R}$ the functions $f(x)=0$ and $f(x)=\cosh (c x)$, where $c \in \mathbb{R}$. Aczél and Dhombres in [1] wrote several chapters covering and connecting the Cauchy, d'Alembert and the trigonometric functional equations.

The domain of definition of the solutions of (1.1) have progressively been extented from $\mathbb{R}$ via abelian groups and monoids (See Aczél and Dhombres [1], Davison [5,6] and Stetkær [24] for details and references), and the functional equation (1.1) take the general forme

$$
f(x y)+\mu(y) f(x \sigma(y))=2 f(x) f(y)
$$

for all $x, y \in S$, where $S$ is a semigroup, $\sigma: S \longrightarrow S$ is an involutive anti-automorphism of $S$, i.e., $\sigma(x y)=\sigma(y) \sigma(x)$ and $\sigma(\sigma(x))=x$ for all $x, y \in S$, and $\mu: S \longrightarrow \mathbb{C}$ is a multiplicative function on $S: \mu(x y)=\mu(x) \mu(y)$ for all $x, y \in S$, which satisfies $\mu(x \sigma(x))=1$ for all $x \in S$. The latest result about the functional equation (1.2) is due to Stetkær [20].

By combining an algebraic small dimension lemma with methods by Davison $[5,6]$ on monoids, Stetkær [20] described the solution of (1.2) on semigroups in terms of multiplicative functions and 2-dimensional irreducible representations, like they have been in previous studies of solutions on groups and monoids $[5,6]$.

Recently, a number of Mathematicians have studied other versions of d'Alembert's functional equations

$$
f(x \sigma(y))+f(\tau(y) x)=2 f(x) g(y)
$$

Key words and phrases. Topological group, monoid, d'Alembert's equation, automorphism, involution. 2020 Mathematics Subject Classification. 39B32. 
for all $x, y \in S$ on smigroups and monoids, and where $\sigma, \tau: S \longrightarrow S$ are respectively involutives anti-automorphisms, resp. $\sigma, \tau: S \longrightarrow S$ are involutives automorphisms. (See [7], [10], [11], [14], [15], [17] and [19]). Since in two situations $\sigma \circ \tau$ is an automorphism, they succefully used some steps and ideas of Stetkær [23] to eliminate $\sigma$ and $\tau$ from the equation (1.3) and brought the equation to a trigonometric equation, and consequently non-abelian phenomena like representations crop up.

In this paper, we solve the functional equation

$$
f(x \sigma(y))+f(\tau(y) x)=2 f(x) f(y)
$$

for all $x, y \in M$, where $M$ is a monoid, $\sigma: M \longrightarrow M$ is an involutive automorphism, and $\tau: M \longrightarrow M$ is involutive anti-automorphism. The crucial idea used in the paper of Stetkær [23] can not carry over to the our situation, in which $\sigma \circ \tau$ is an anti-autmorphism of $M$. The functional equation (1.4) is a natural generalization of the variant of Wilson's functional equation equation

$$
f(x y)+f\left(y^{-1} x\right)=2 f(x) g(y), x, y \in G
$$

studied by Stetkær [26] and solved by Ebanks and Stetkær [8] on groups.

Davison in [5], [6, Theorem 4.12] and Stetkær [24, Theorem 8.26] obtained continuous solutions of the pre-d'Alembert functional equation

$$
g(x y z)+g(x z y)=2 g(x) g(y z)+2 g(y) g(x z)+2 g(x y) g(z)-4 g(x) g(y) g(z)
$$

for all $x, y, z \in M$ on a topological groups and monoids.

In Proposition 3.2 and Proposition 3.3 we prove some properties of the solutions of our functional equation (1.4). In particular, the solutions are central and satisfies the pre-d'Alembert functional equation (Proposition 3.3). By using this result we give a full description of functions $f: M \rightarrow \mathbb{C}$ satisfying the equation (1.4) in terms of multiplicative functions, and characters of 2-dimensional irreducible representations of $M$. Thus functional equation (1.4) makes sense to solve other functional equations by expressing their solutions in terms of solutions of (1.4). This is what we shall do for generalized of variant of Van Vleck's

$$
f\left(y^{-1} x z_{0}\right)-f\left(x \sigma(y) z_{0}\right)=2 f(x) f(y), \quad x, y \in G .
$$

and for generalized of variant Kannappan's functional equation

$$
f\left(x \sigma(y) z_{0}\right)+f\left(\tau(y) x z_{0}\right)=2 f(x) f(y), \quad x, y \in M,
$$

where $z_{0}$ is a fixed element in $M$. Some information, applications and numerous references concerning Van Vleck's and Kannappan's functional equation and their further generalizations can be found e.g. in [3], [12], [13], [18] and [22]- [30].

\section{Set Up and Notation}

Throughout this paper $S$ designed a semigroup, $M$ a monoid, and $G$ a group with neutral element $e$. The map $\sigma: M \rightarrow M$ denotes an involutive homomorphism and the map $\tau$ an involutive anti-automorphism. That $\sigma, \tau: M \rightarrow M$ are involutive means that $\tau(\tau(x))=$ $\sigma(\sigma(x))=x$ for all $x \in M$. We need the following basic definitions.

Definition 2.1. (1) A function $f: S \rightarrow \mathbb{C}$ is central provided that $f(x y)=f(y x)$ for all $x, y \in S$. 
(2) A function $f: S \rightarrow \mathbb{C}$ is abelian means

$$
f\left(x_{1} x_{2} \ldots x_{n}\right)=f\left(x_{\epsilon(1)} x_{\epsilon(2)} \ldots x_{\epsilon(n)}\right)
$$

for every $n \geq 2$ and every permutation $\epsilon$ of $n$ elements of $S$.

(3) A function $f: S \rightarrow \mathbb{C}$ is $\sigma$-even if $f(\sigma(x))=f(x)$ for all $x \in S$, and $\sigma$-odd if $f(\sigma(x))=-f(x)$ for all $x \in S$.

(4) A function $\chi: S \longrightarrow \mathbb{C}$ is said to be multiplicative if $\chi(x y)=\chi(x) \chi(y)$ for all $x, y \in S$.

For any vector space $V$, we let $\mathcal{L}(V)$ denote the algebra of linear operators of $V$ into $V$. A representation of $M$ on non-zero vector space $V$ is a map $\pi: M \rightarrow \mathcal{L}(V)$ such that $\pi(x y)=\pi(x) \pi(y)$ for all $x, y \in M$. The space $V$ is called the representation space of $\pi$. We furthermore assume that $\pi(e)=I$ : The identity map.

Let $\operatorname{dim} V<+\infty$. The dimension of the representation $\pi$ is $d_{\pi}=\operatorname{dim} V$ and the matrix coefficient $\chi_{\pi}: M \rightarrow \mathbb{C}$ defined by $\chi_{\pi}(x):=\operatorname{tr}(\pi(x)), x \in M$ is called the character of $\pi$. If $M$ is a topological space, then we let $C(M)$ denote the algebra of continuous functions from $M$ into $\mathbb{C}$.

Let $V$ be a 2-dimensional vector space. To any linear map $A \in \mathcal{L}(V)$ we define its adjugate $\operatorname{adj}(A) \in \mathcal{L}(V)$ from linear algebra. The properties of adjugation can easily be derived from its matrix form, and in particular we have $a d j: \mathcal{L}(V) \rightarrow \mathcal{L}(V)$ is linear,

$$
A+\operatorname{adj}(A)=(\operatorname{tr} A) I .
$$

Furthermore, $\operatorname{Aadj}(A)=\operatorname{adj}(A) A=(\operatorname{det} A) I, \operatorname{adj}(A B)=\operatorname{adj}(B) \operatorname{adj}(A), \operatorname{and} \operatorname{adj}(\operatorname{adj}(A))=$ $A$ for all $A, B \in \mathcal{L}(V)$.

\section{Some properties of equation (1.4)}

In this section, we prove some properties of the functional equation (1.4) on semigroups and monoids. In particular, we prove that solutions of of equation (1.4) are solutions of pre-d'Alembert functional equation.

Lemma 3.1. Let $f: M \rightarrow \mathbb{C}$ be a non-zero solution of the functional equation (1.4). Then

$$
\left\{\begin{array}{l}
f(e)=1 \\
f(\sigma(x))+f(\tau(x))=2 f(x)
\end{array}\right.
$$

Proof. Take $y=e$ in (1.4) we get $f(x)=f(e) f(x)$. Since $f \neq 0$ then $f(e)=1$.

Putting $x=e$ in (1.4) we get the following identity

$$
f(\sigma(y))+f(\tau(y))=2 f(e) f(y) .
$$

Using that $f(e)=1$ we get

$$
f(\sigma(y))+f(\tau(y))=2 f(y)
$$

for all $y \in M$. In the following proposition we eliminate the involution anti-automorphism $\tau$ from the functional equation (1.4). 
Proposition 3.2. Let $f: S \rightarrow \mathbb{C}$ be a non-zero solution of the functional equation (1.4). Then

$f(\sigma(a) \sigma(x) \sigma(y))+f(\sigma(a) \sigma(y) \sigma(x))=2 f(\sigma(a)) f(y x)+2 f(x) f(\sigma(a) \sigma(y))+2 f(\sigma(a) \sigma(x)) f(y)-4 f(\sigma(a)) f(x) f(y)$,

for all $a, x, y \in S$.

Proof. For the pair $(\sigma(a) \sigma(x), y)$ the functional equation (1.4 ) can be written as follows

$$
f(\sigma(a) \sigma(x) \sigma(y))+f(\tau(y) \sigma(a) \sigma(x))=2 f(\sigma(a) \sigma(x)) f(y) .
$$

By applying (1.4) to the pair $(\tau(y) \sigma(a), x)$ we obtain

$f(\tau(y) \sigma(a) \sigma(x))+f(\tau(x) \tau(y) \sigma(a))=2 f(\tau(y) \sigma(a)) f(x)=2 f(x)[2 f(\sigma(a)) f(y)-f(\sigma(a) \sigma(y))]$.

By using (1.4), we reformulate the second term on the left hand side of (3.4) as follows

$$
f(\tau(x) \tau(y) \sigma(a))=f(\tau(y x) \sigma(a))=2 f(\sigma(a)) f(y x)-f(\sigma(a) \sigma(y x)),
$$

which turns the identity (3.4) into we obtain

$f(\tau(y) \sigma(a) \sigma(x))+2 f(\sigma(a)) f(y x)-f(\sigma(a) \sigma(y) \sigma(x))=4 f(\sigma(a)) f(x) f(y)-2 f(x) f(\sigma(a) \sigma(y))$.

Subtracting this from (3.3) we get after some simplifications that

$f(\sigma(a) \sigma(x) \sigma(y))+f(\sigma(a) \sigma(y) \sigma(x))=2 f(\sigma(a)) f(y x)+2 f(x) f(\sigma(a) \sigma(y))+2 f(\sigma(a) \sigma(x)) f(y)-4 f(\sigma(a)) f(x) f(y)$

for all $a, x, y \in S$. This ends the proof.

Proposition 3.3. Let $f: S \rightarrow \mathbb{C}$ be a non-zero solution of the functional equation (1.4). Then

(1) $f$ is central.

(2) $f(\sigma(x))+f(\tau(x))=\lambda f(x)$ for all $x \in S$, and for same $\lambda \in \mathbb{C}^{*}$.

(3) $f$ is a solution of the pre-d'Alembert functional equation i.e.,

$f(a x y)+f(a y x)=2 f(a) f(x y)+2 f(x) f(a y)+2 f(a x) f(y)-4 f(a) f(x) f(y)$

for all $a, x, y \in S$.

Proof. (1) According to Proposition $3.2 f$ satisfies (3.2). Interchange of $x$ and $y$ in (3.2) and comparing the result obtained with (3.2) we find that $f(\sigma(a)) f(x y)=$ $f(\sigma(a)) f(y x)$ for all $a, x, y \in S$. Since $f \neq 0$, then $f$ is central.

(2) Replace $x$ by $\sigma(x)$ in (1.4), we obtain

$$
f(\sigma(x) \sigma(y))+f(\tau(y) \sigma(x))=2 f(\sigma(x)) f(y), \quad x, y \in S .
$$

Substitute $x$ by $\tau(x)$ in in (1.4), we obtain

$$
f(\tau(x) \sigma(y))+f(\tau(y) \tau(x))=2 f(\tau(x)) f(y), \quad x, y \in S .
$$

Adding (3.6) and (3.7) and use that $f$ is central we obtain

$$
\begin{gathered}
{[f(\sigma(y) \sigma(x))+f(\tau(x) \sigma(y))]+[f(\tau(y) \sigma(x))+f(\tau(x) \tau(y))]} \\
\quad=f(x)(f(\sigma(y))+f(\tau(y)))=f(y)(f(\sigma(x))+f(\tau(x)))
\end{gathered}
$$


for all $x, y \in S$. Since $f \neq 0$ then there exists $y_{0} \in S$ such that $f\left(y_{0}\right) \neq 0$, and that $f(\sigma(x))+f(\tau(x))=\lambda f(x)$ for all $x \in S$, and where $\lambda=\frac{f\left(\sigma\left(y_{0}\right)\right)+f\left(\tau\left(y_{0}\right)\right)}{f\left(y_{0}\right)}$.

Now, if $\lambda=0$, we get $f(\sigma(x))=-f(\tau(x))$ for all $x \in S$. By replacing $x$ by $\sigma(x), y$ by $\tau(y)$ respectively $x$ by $\tau(x)$ and $y$ by $\sigma(y)$ in (1.4) we get

$$
\left\{\begin{array}{l}
f(\sigma(x) \sigma(\tau(y)))+f(y \sigma(x))=2 f(\sigma(x)) f(\tau(y)) \\
f(\tau(x) y)+f(\tau(\sigma(y)) \tau(x))=2 f(\tau(x)) f(\sigma(y))
\end{array}\right.
$$

Since $f(\sigma(x))=-f(\tau(x)), \quad x \in S$ and $f$ is central, so by adding the two functional equations of the above system we get

$$
-f(\tau(x) y)-f(y \sigma(x))+f(y \sigma(x))+f(\tau(x) y)=-4 f(\sigma(x)) f(\sigma(y))=0
$$

for all $x, y \in S$. Since $\sigma^{2}=I$, so $f=0$, which is a contradiction with $f \neq 0$.

(3) Let $a, x, y \in S$ be arbitrary. For the pair $(\tau(x) \tau(a), y)$ equation (1.4 implies that

$$
f(\tau(x) \tau(a) \sigma(y))+f(\tau(y) \tau(x) \tau(a))=2 f(\tau(x) \tau(a)) f(y) .
$$

By applying (1.4) to the pair $(\tau(a) \sigma(y), x)$ we obtain

$$
f(\tau(a) \sigma(y) \sigma(x))+f(\tau(x) \tau(a) \sigma(y))=2 f(\tau(a) \sigma(y)) f(x)=2 f(x)[2 f(\tau(a)) f(y)-f(\tau(y) \tau(a))] .
$$

By using (1.4) we reformulate the first term on the left hand side of (3.9) as follows

$$
f(\tau(a) \sigma(y x))=2 f(\tau(a)) f(y x)-f(\tau(x) \tau(y) \tau(a)),
$$

which turns the identity (3.9) into

$2 f(\tau(a)) f(y x)-f(\tau(x) \tau(y) \tau(a))+f(\tau(x) \tau(a) \sigma(y))=4 f(\tau(a)) f(x) f(y)-2 f(x) f(\tau(y) \tau(a))$.

Subtracting this from (3.8) we get after some simplifications that

$f(\tau(a x y))+f(\tau(a y x))=2 f(\tau(a)) f(y x)+2 f(x) f(\tau(a y))+2 f(\tau(a x)) f(y)-4 f(\tau(a)) f(x) f(y)$

By adding (3.2) and (3.10), and using Proposition 3.3 (2), we conclude that $f$ is a solution of pre-d'Alembert functional equation on semigroup $S$. This completes the proof.

Now, we are ready to prove the main result.

Theorem 3.4. Abelian solutions of equation (1.4).

The abelian solutions $f: M \rightarrow \mathbb{C}$ of (1.4) are the functions of the form

$$
f=\frac{\chi+\chi \circ \sigma \circ \tau}{2}
$$

where $\chi: M \rightarrow \mathbb{C}$ is a multiplicative function such that:

(i) $\chi \circ \sigma \circ \tau=\chi \circ \tau \circ \sigma$, and

(ii) $\chi$ is $\sigma$-even and/or $\tau$-even.

Proof. It is elementary to check that the functions stated in the Theorem 3.4 define abelian solutions, so it is left to show that any abelian solution $f: M \rightarrow \mathbb{C}$ of (1.4) can be written as in the form in Theorem 3.4. We have from proposition 3.3 that $f$ is solution of pred'Alembert functional equation on a Monoid $M$, and which satisfies $f(e)=1$, (see Lemma 
3.1). If $f$ is an abelian solution by [24, Theorem 8.13.] then there exist multiplicative functions $\chi_{1}, \chi_{2}: M \rightarrow \mathbb{C}$ such that $f=\frac{\chi_{1}+\chi_{2}}{2}$. We will discuss two cases.

Case 1. If $\chi_{1}=\chi_{2}$, then letting $\chi:=\chi_{1}=\chi_{2}$ we have $f=\chi$. Substituting $f=\chi$ into (1.4) we get that $\chi \circ \sigma+\chi \circ \tau=2 \chi$. So $\chi=\chi \circ \sigma=\chi \circ \tau$. Then $f$ has the desired form.

Case 2. If $\chi_{1} \neq \chi_{2}$, substituting $f=\left(\chi_{1}+\chi_{2}\right) / 2$ into (1.4) we find after a reduction that

$$
\begin{aligned}
& \chi_{1}(x)\left[\chi_{1}(\sigma(y))+\chi_{1}(\tau(y))-\chi_{1}(y)-\chi_{2}(y)\right] \\
& \left.+\chi_{2}(x)\left[\chi_{2}(\sigma(y))+\chi_{2}(\tau(y))-\chi_{1}(y)-\chi_{2}(y)\right]\right]=0
\end{aligned}
$$

for all $x, y \in M$. Since $\chi_{1} \neq \chi_{2}$, we get from the theory of multiplicative functions (see for instance ( $[24$, Theorem 3.18]) that both terms are 0 , so

$$
\left\{\begin{array}{l}
\chi_{1}(x)\left[\chi_{1}(\sigma(y))+\chi_{1}(\tau(y))-\chi_{1}(y)-\chi_{2}(y)\right]=0 \\
\chi_{2}(x)\left[\chi_{2}(\sigma(y))+\chi_{2}(\tau(y))-\chi_{1}(y)-\chi_{2}(y)\right]=0
\end{array}\right.
$$

for all $x, y \in M$. Since $\chi_{1} \neq \chi_{2}$ at least one of $\chi_{1}$ and $\chi_{2}$ is not zero.

Now if $\chi_{1} \neq 0$ and $\chi_{2}=0$. It is allowed that $\chi_{1}(\sigma(y))+\chi_{1}(\tau(y))=\chi_{1}(y)$ this implies that $\chi_{1}(\sigma(y))=0$ or $\chi_{1}(\tau(y))=0$, in either case $\chi_{1}=0$ because $\sigma^{2}=\tau^{2}=i d$.

We have now $\chi_{1} \neq 0$ and $\chi_{2} \neq 0$. From (3.11), we get

$$
\chi_{1}+\chi_{2}=\chi_{1} \sigma+\chi_{1} \circ \tau=\chi_{2} \circ \sigma+\chi_{2} \circ \tau .
$$

Using that $\chi_{1} \neq \chi_{2}$, we obtain that $\chi_{2}=\chi_{1} \circ \sigma \circ \tau=\chi_{1} \circ \tau \circ \sigma$ now as we see that $\chi_{1}+\chi_{2}=\chi_{1} \sigma+\chi_{1} \circ \tau$ this implies that $\chi_{1}$ is is $\sigma$-even or $\tau$-even. Finally, we deduce that $f$ has the form stated in Theorem 3.4 with $\chi=\chi_{1}$.

Theorem 3.5. Non-abelian solutions of equation (1.4).

The non-abelian solutions $f M \longrightarrow \mathbb{C}$ of (1.4) are of the form $f(x)=\frac{1}{2} \operatorname{tr} \pi(x)$ for all $x \in M$, where $\pi$ is an irreducible, 2-dimensional representation of $M$ for which $\operatorname{adj}(\pi \circ \sigma)=\pi \circ \tau$, $\operatorname{tr}(\pi(x))=\operatorname{tr}(\pi(\sigma(x)))=\operatorname{tr}(\pi(\tau(x)))=\operatorname{tr}(\pi(\sigma \circ \tau(x)))=\operatorname{tr}(\pi(\tau \circ \sigma(x)))$, for all $x \in M$.

If $\pi^{\prime}$ is any irreducible representation of $M$ on a finite dimensional vector space, such that $f(x)=\frac{1}{2} \operatorname{tr} \pi^{\prime}(x)$ for all $x \in M$, then $\pi^{\prime}$ and $\pi$ are equivalent.

Proof. By Proposition 3.3 (3), $f$ is a non-abelian solution of the pre-d'Alembert functional equation on $M$. Then from [24, Theorem 8.26] $f$ has the form $f=\frac{1}{2} \operatorname{tr}(\pi)$, where $\pi$ is an irreducible, 2-dimensional representation of $M$. Substituting $f$ into the functional equation (1.4) and using that $f$ is central we get

$$
\operatorname{tr}(\pi(x)(\pi(\sigma(y)+\pi(\tau(y))-\operatorname{tr}(\pi(y)) I)))=0, \quad x, y \in M
$$

Now, Burnside Theorem's [16] shows that

$$
\operatorname{tr}(A(\pi(\sigma(y)+\pi(\tau(y))-\operatorname{tr}(\pi(y)) I))=0,
$$

for all $A \in \mathbf{L}(V)$ and for all $y \in M$, and consequently, we get

$$
\pi\left(\sigma(y)+\pi(\tau(y))=\operatorname{tr}(\pi(y)) I_{2}\right.
$$


for all $y \in M$. Replacing $y$ by $\sigma(y)$ respectively by $\tau(y)$ in (3.12) we obtain

$$
\left\{\begin{array}{l}
\pi(y)+\pi(\tau(\sigma(y)))=\operatorname{tr}(\pi(\sigma(y))) I_{2} \\
\pi(y)+\pi(\sigma(\tau(y)))=\operatorname{tr}(\pi(\tau(y))) I_{2}
\end{array}\right.
$$

By adding the two functional equation of the above system and using that $f(\sigma(y))+$ $f(\tau(y))=2 f(y)$ for all $y \in M$, we obtain

$$
\pi(y)+\frac{\pi(\tau(\sigma(y)))+\pi(\sigma(\tau(y)))}{2}=\operatorname{tr}(\pi(y)) I_{2},
$$

for all $y \in M$. From the properties of adjugation (2.1) we get $\operatorname{adj}(\pi(y))=\frac{\pi(\tau(\sigma(y)))+\pi(\sigma(\tau(y)))}{2}$, for all $y \in M$. Since $\operatorname{adj}(\pi(x y))=\operatorname{adj}(\pi(x) \pi(y))=\operatorname{adj}(\pi(y)) \operatorname{adj}(\pi(x))$ then

$$
\pi(\tau(\sigma(y)))(\pi(\tau(\sigma(x)))-\pi(\sigma(\tau(x))))=\pi(\sigma(\tau(y)))(\pi(\tau(\sigma(x)))-\pi(\sigma(\tau(x)))), \quad x, y \in M .
$$

Now by using (3.13) we get

$$
(\pi(\tau(\sigma(y)))-\pi(\sigma(\tau(y))))=(\operatorname{tr}(\pi(\sigma(y)))-\operatorname{tr}(\pi(\tau(y)))) I_{2}, \quad y \in M
$$

since (3.14) then $\operatorname{adj}(\pi(y))=\pi(\tau(\sigma(y)))=\pi(\sigma(\tau(y)))$, this implies that $\operatorname{adj}(\pi(\sigma(y)))=$ $\pi(\tau(y))$. By the condition $f(\sigma(y))+f(\tau(y))=2 f(y)$ we see that $\operatorname{tr}(\pi(\sigma(y)))+\operatorname{tr}(\pi(\tau(y)))=$ $2 \operatorname{tr}(\pi(y)$, so we get $\operatorname{tr}(\pi(\sigma(y)))=\operatorname{tr}(\pi(y)$. Finally we obtain the desired non-abelian solution.

Remark 3.6. By using similar proof (see Proposition 3.3), we show the centrality of the solutions of the functional equation (1.4), when $\sigma$ is an involutive anti-automorphism and $\tau$ is in involutive automorphism. So, the order of $\sigma$ and $\tau$ in the functional equation (1.4) is not important.

The following corollaries are immediate consequences of Theorem 3.4 and Theorem 3.5.

Corollary 3.7. Let $G$ be a topological group and $\sigma: G \longrightarrow G$ be a continuous involutive automorphism of $G$. There are two non-zero solutions $f: G \rightarrow \mathbb{C}$ of the functional equation

$$
f(x \sigma(y))+f\left(y^{-1} x\right)=2 f(x) f(y), x, y \in G,
$$

abelian and non-abelian:

(1) Every abelian continuous solution $f$ is of the form

$$
f(x)=\frac{\chi(x)+\chi\left(\sigma\left(x^{-1}\right)\right.}{2},
$$

where $\chi: G \rightarrow \mathbb{C}$ is a continuous multiplicative function such that $\chi$ is $\sigma$-even and/or $\chi(x)=\chi\left(x^{-1}\right)$.

(2) Every non-abelian continuous solution $f$ is of the form

$$
f(x)=\frac{1}{2} \operatorname{tr} \pi(x), \quad x \in G,
$$

where $\pi$ is a continuous irreducible unitary representation of dimension 2 for which $\operatorname{adj}(\pi(\sigma(x)))=\pi\left(x^{-1}\right)$, and $\operatorname{tr}(\pi(\sigma(x)))=\operatorname{tr}(\pi(x))$.

Proof. It suffices to take $\tau(x)=x^{-1}$ for all $x \in G$ in Theorem 3.4 and Theorem 3.5. 
Corollary 3.8. Let $M$ be a topological monoid and $\tau$ be a continuous involutive antiautomorphism of $M$. There are two non-zero continuous solutions $f: M \rightarrow \mathbb{C}$ of the functional equation

$$
f(x y)+f(\tau(y) x)=2 f(x) f(y), x, y \in G,
$$

abelian and non-abelian:

(1) Every abelian continuous solution $f$ is of the form

$$
f=\frac{\chi+\chi \circ \tau}{2},
$$

where $\chi: M \rightarrow \mathbb{C}$ is a continuous multiplicative function.

(2) Every non-abelian continuous solution $f$ is of the form

$$
f(x)=\frac{1}{2} \operatorname{tr} \pi(x), \quad x \in M,
$$

where $\pi$ is a continuous irreducible unitary representation of dimension 2 for which $\operatorname{adj}(\pi)=\pi \circ \tau$.

Proof. It suffices to take $\sigma(x)=x$ for all $x \in M$ in Theorem 3.4 and Theorem 3.5.

Corollary 3.9. Let $G$ be a topological group. There are two non-zero continuous solutions $f: G \rightarrow \mathbb{C}$ of the functional equation

$$
f(x y)+f\left(y^{-1} x\right)=2 f(x) f(y), x, y \in G,
$$

abelian and non-abelian:

(1) Every abelian continuous solution $f$ is of the form

$$
f(x)=\frac{\chi(x)+\chi\left(x^{-1}\right)}{2}, x \in G
$$

where $\chi: G \rightarrow \mathbb{C}^{*}$ is a continuous multiplicative function.

(2) Every non-abelian continuous solution $f$ is of the form

$$
f(x)=\frac{1}{2} \operatorname{tr} \pi(x), \quad x \in G,
$$

where $\pi$ is a continuous irreducible unitary representation of dimension 2 for which $\operatorname{adj}(\pi(x))=\pi\left(x^{-1}\right)$.

Proof. It suffices to take $\sigma(x)=x$ and $\tau(x)=x^{-1}$ for all $x \in G$ in Theorem 3.4 and Theorem 3.5.

\section{Some Applications And EXamples}

In this section, we determine solutions of the functional equations (1.6) and (1.7). We show that the solutions are closely related to the solutions of (1.4).

Proposition 4.1. Let $z_{0}$ in $Z(G)$ the center of $G$ be given. Let $f: G \rightarrow \mathbb{C}$ be a non-zero solution of the functional equation (1.6). Then

(1) $f(e)=0$.

(2) $f\left(y^{-1} z_{0}\right)=f\left(\sigma(y) z_{0}\right), y \in G$.

(3) $f\left(y^{-1}\right)=-f(\sigma(y)), \quad y \in G$. 
(4) $f\left(y \sigma\left(z_{0}\right) z_{0}\right)=-f(y), y \in G$.

(5) $f\left(\sigma\left(z_{0}\right) z_{0}\right)=0$.

(6) $f\left(z_{0}\right)=1$.

(7) $f\left(z_{0}^{2}\right)=0$.

Proof. (1) Substituting $y=e$ into (1.6) and using that $f \neq 0$, we get that $f(e)=0$.

(2) Take $x=e$ in (1.6) and using that $f(e)=0$ we obtain $f\left(y^{-1} z_{0}\right)=f\left(\sigma(y) z_{0}\right)$ for all $y \in G$.

(3) Replacing $x$ by $\sigma\left(x^{-1}\right)$ in (1.6) we get

$$
f\left(y^{-1} \sigma\left(x^{-1}\right) z_{0}\right)-f\left(\sigma\left(x^{-1}\right) \sigma(y) z_{0}\right)=2 f\left(\sigma\left(x^{-1}\right)\right) f(y), \quad x, y \in G .
$$

This implies that

$$
f\left((\sigma(x) y)^{-1} z_{0}\right)-f\left(\left(\sigma\left(y^{-1}\right) \sigma(x)\right)^{-1} z_{0}\right)=2 f\left(\sigma\left(x^{-1}\right)\right) f(y), \quad x, y \in G .
$$

Using (2) we get

$$
f\left(x \sigma(y) z_{0}\right)-f\left(y^{-1} x z_{0}\right)=2 f\left(\sigma\left(x^{-1}\right)\right) f(y), \quad x, y \in G .
$$

From (1.6) and that $f \neq 0$ we obtain $f\left(x^{-1}\right)=-f(\sigma(x))$ for all $x \in G$.

(4) Taking $y=\sigma(x) z_{0}$ into (2) and using that $z_{0} \in Z(G)$ we get $f\left(\sigma\left(x^{-1}\right)\right)=f\left(x \sigma\left(z_{0}\right) z_{0}\right)$ since $f\left(x^{-1}\right)=-f(\sigma(x))$, then $f\left(x \sigma\left(z_{0}\right) z_{0}\right)=-f(x)$.

(5) Putting $y=z_{0}$ in (2) we get $0=f(e)=f\left(\sigma\left(z_{0}\right) z_{0}\right)$.

(6) Replacing $y$ by $z_{0}$ in (1.6) and using $z_{0} \in Z(G)$ we have $f(x)-f\left(x \sigma\left(z_{0}\right) z_{0}\right)=$ $2 f(x) f\left(z_{0}\right)$, since $f\left(x \sigma\left(z_{0}\right) z_{0}\right)=-f(x)$, so $2 f(x)=2 f(x) f\left(z_{0}\right)$, but $f \neq 0$ then $f\left(z_{0}\right)=1$.

(7) Replacing $y$ by $z_{0}^{-1}$ in (2) and using (3) we obtain $f\left(z_{0}^{2}\right)=f\left(\sigma\left(z_{0}^{-1}\right) z_{0}\right)=f\left(\sigma\left(z_{0}^{-1} \sigma\left(z_{0}\right)\right)=\right.$ $-f\left(\sigma\left(z_{0}\right)^{-1} z_{0}\right)$ then $f\left(z_{0}^{2}\right)=0$

Lemma 4.2. Let $z_{0}$ in $Z(G)$ be given. Let $f: G \rightarrow \mathbb{C}$ be a non-zero solution of the functional equation (1.6). Then

(1) $f\left(z_{0}^{-1}\right)^{2}=f\left(\sigma\left(z_{0}\right)\right)^{2}=1$.

(2) $f\left(z_{0}^{3}\right)=f\left(z_{0}^{-1}\right)=-f\left(\sigma\left(z_{0}\right)\right)$.

Proof. (1) If we take $x=y=z_{0}^{-1}$ in (1.6) and using $z_{0} \in Z(G)$ we get

$$
f\left(z_{0}\right)-f\left(\sigma\left(\left(z_{0}\right)^{-1}\right)\right)=2 f\left(\left(z_{0}\right)^{-1}\right)^{2}
$$

Using that $f\left(z_{0}\right)=1$ and $f\left(y^{-1}\right)=-f(\sigma(y)), y \in G$, we get $f\left(z_{0}^{-1}\right)^{2}=f\left(\sigma\left(z_{0}\right)\right)^{2}=$ 1 .

(2) Replacing $y$ by $\left(\left(z_{0}\right)^{2}\right)^{-1}$ in Proposition 4.3 (2) and using and Proposition 4.3 (4) we get

$$
f\left(z_{0}^{3}\right)=f\left(\sigma\left(z_{0}\right)^{2} z_{0}\right)=f\left(\sigma\left(z_{0}\right) \sigma\left(z_{0}\right) z_{0}\right)=-f\left(\sigma\left(z_{0}\right)\right) .
$$

This ends the proof.

Theorem 4.3. Let $G$ be a topological group and let $z_{0} \in Z(G)$ be given. Let $f: G \rightarrow \mathbb{C}$ be a continuous solution of the functional equation (1.6).

(1) If $\sigma\left(z_{0}\right) z_{0}=e$, then (1.6) has no non-zero solutions.

(2) $\sigma\left(z_{0}\right) z_{0} \neq e$, then the solutions have the following forms 
(a) $f(x)=\frac{\chi(x)-\chi\left(x^{-1}\right)}{2 \chi\left(z_{0}\right)}, \quad x \in G$, where $\chi: G \rightarrow \mathbb{C}^{*}$ is a continuous multiplicative function such that $\chi(\sigma(x))=\chi(x)$ and $\chi\left(z_{0}\right)^{2}=-1$.

(b) $f(x)=\frac{\chi(x)-\chi(\sigma(x))}{2 \chi\left(z_{0}\right)}$, where $\chi: G \rightarrow \mathbb{C}^{*}$ is a continuous multiplicative function such that $\chi(x)=\chi\left(x^{-1}\right)$ and $\chi\left(\sigma\left(z_{0}\right) z_{0}\right)=-1$.

Proof. (1) If $\sigma\left(z_{0}\right) z_{0}=e$, by Proposition 4.1 (4) $f\left(x \sigma\left(z_{0}\right) z_{0}\right)=-f(x), \quad x \in G$, so we have $f(x)=0$ for all $x \in G$.

(2) From now, we assume that $\sigma\left(z_{0}\right) z_{0} \neq e$. Replacing $x$ by $x z_{0}$ and $y$ by $y z_{0}$ in (1.6) and using that $z_{0} \in Z(G)$ we get

$$
f\left(y^{-1} x z_{0}\right)-f\left(x \sigma(y) z_{0} \sigma\left(z_{0}\right) z_{0}\right)=2 f\left(x z_{0}\right) f\left(y z_{0}\right)
$$

since $f\left(x \sigma\left(z_{0}\right) z_{0}\right)=-f(x), y \in G$, then

$$
f\left(y^{-1} x z_{0}\right)+f\left(x \sigma(y) z_{0}\right)=2 f\left(x z_{0}\right) f\left(y z_{0}\right), \quad x, y \in G .
$$

Consider $g(x)=f\left(x z_{0}\right), \quad x \in G$, then the function $g$ satisfies (1.4) with $\tau(x)=x^{-1}$ and $g(e)=1$, so $g \neq 0$. As $g$ is a solution of equation (1.4) then by Proposition $3.3(3) \mathrm{g}$ is a solution of pre-d'Alembert functional equation. From Proposition 4.1 (7) we get $g\left(z_{0}\right)=0$ and by Lemma 4.2 we have $d\left(z_{0}\right)=2 g\left(z_{0}\right)^{2}-g\left(z_{0}^{2}\right)=$ $0-f\left(z_{0}^{3}\right)=0-\left(-f\left(\sigma\left(z_{0}\right)\right)=f\left(\sigma\left(z_{0}\right)\right) \neq 0\right.$. So, we get $g\left(z_{0}\right)^{2} \neq d\left(z_{0}\right)$. According to [24, Proposition 8.14(a)] we have $g$ is abelian. Furthermore, from Corollary 3.6 (1) $g(x)=\frac{\chi(x)+\chi\left(\sigma\left(x^{-1}\right)\right)}{2}$ for all $x \in G$, where $\chi$ is a non-zero continuous multiplicative function such that $\chi$ is $\sigma$-even and/or $\chi(x)=\chi\left(x^{-1}\right)$. Since $g\left(z_{0}\right)=0$, so $\chi\left(z_{0}\right)=$ $-\chi\left(\sigma\left(z_{0}^{-1}\right)\right)$, which implies that $\chi\left(z_{0} \sigma\left(z_{0}\right)\right)=-1$. We have $f(x)=g\left(x z_{0}^{-1}\right)$ and $\chi\left(z_{0} \sigma\left(z_{0}\right)\right)=-1$, so we get that $f(x)=\frac{\chi(x)-\chi\left(\sigma\left(x^{-1}\right)\right)}{2 \chi\left(z_{0}\right)}$. This completes the proof.

Theorem 4.4. Let $M$ be a topological monoid. Let $z_{0}$ be an arbitrarily fixed element in $M$. There are two non-zero continuous solutions $f: G \rightarrow \mathbb{C}$ of (1.7), abelian and non-abelian.

(1) Every abelian continuous solution $f$ is of the form

$$
f=f(e) \frac{\chi+\chi \circ \sigma \circ \tau}{2},
$$

where $\chi: G \rightarrow \mathbb{C}$ is a continuous multiplicative function such that:

(i) $\chi \circ \sigma \circ \tau=\chi \circ \tau \circ \sigma$,

(ii) $\chi$ is $\sigma$-even and/or $\tau$-even, and

(iii) $\chi\left(\sigma\left(\tau\left(z_{0}\right)\right)\right)=\chi\left(z_{0}\right)=f(e)$.

(2) Every non-abelian continous solution $f$ is of the form

$$
f(x)=\frac{f(e) \chi_{\pi}(x)}{2}, \quad x \in G
$$

where $\pi$ is a continuous irreducible representation of dimension 2 for which adj $(\pi \circ$ $\sigma)=\pi \circ \tau, \operatorname{tr}(\pi(x))=\operatorname{tr}(\pi(\sigma(x))), \quad x \in M$, and $\pi\left(z_{0}\right)=f(e) I_{2}$

Proof. Taking $y=e$ in (1.7), we get that

$$
f\left(x z_{0}\right)=f(x) f(e), x \in M .
$$


We have $f \neq 0$, so $f(e) \neq 0$. Using (1.7) and (4.2) twe get that the function $g(x)=\frac{f(x)}{f\left(z_{0}\right)}$ is a solution of (1.4). Therefore, by Theorem 3.4, Theorem 3.5 and the identity (4.2) it is easy to get the form of solutions of (1.7).

Conversely, It is easy to check that the all of the possibilities listed in Theorem 4.4 define solutions of (1.7).

Example 4.5. For a non-abelian example of Monoid, we consider $G=M(2, \mathbb{C})$ and take $\sigma$ as an automorphism and $\tau$ as an anti-automorphism such that

$$
\sigma\left(\begin{array}{ll}
a & b \\
c & d
\end{array}\right)=J\left(\begin{array}{ll}
a & b \\
c & d
\end{array}\right) J=\left(\begin{array}{cc}
d & c \\
b & a
\end{array}\right)
$$

where $J=\left(\begin{array}{cc}0 & 1 \\ 1 & 0\end{array}\right)$ and

$$
\tau\left(\begin{array}{ll}
a & b \\
c & d
\end{array}\right)=\left(\begin{array}{cc}
a & -b \\
-c & d
\end{array}\right)
$$

We indicate here the corresponding continuous solutions of (1.4). Simple computations show that

Note that

\section{Abelian solutions.}

$$
\sigma \circ \tau=\tau \circ \sigma
$$

The continuous non-zero multiplicative functions on $M(2, C)$ are given (see [9, Example $5.6]): \mu=1$, or else

$$
\left\{\begin{array}{lr}
\chi(T)=|\operatorname{det}(T)|^{\lambda-n}(\operatorname{det}(T))^{n} & \text { when } \operatorname{det}(T) \neq 0 \\
0 & \text { when } \operatorname{det}(T)=0,
\end{array}\right.
$$

where $\lambda \in \mathbb{C}$ with $\operatorname{Re} \lambda>0$ and $n \in \mathbb{Z}$. It is clear that $\chi(\sigma(\tau(T)))=\chi(\tau(\sigma(T)))$, $\chi(\sigma(T))=\chi(\tau(T))=\chi(T)$. It follows that the abelian continuous solutions are $f(T)=$ $|a d-b c|^{\lambda-n}(a d-b c)^{n}$.

\section{Non-abelian solutions.}

It is clear that $\operatorname{tr}(T)=\operatorname{tr}(\sigma(T)), \operatorname{Adj}(\sigma(T))=\tau(T)$ and $\sigma \circ \tau(T)=\tau \circ \sigma(T)$. By using Theorem 3.4, the non-abelian non-zero continuous solutions $f: M(2, \mathbb{C}) \rightarrow \mathbb{C}$ of $(3.3)$ are $f=\frac{1}{2} t r$.

Example 4.6. The 3-dimension Heisenberg group $G=H_{3}$ described in [24, Example A.17(a)], and take as the involutive automorphism (see [9])

$$
\sigma\left(\begin{array}{ccc}
1 & a & c \\
0 & 1 & b \\
0 & 0 & 1
\end{array}\right)=\left(\begin{array}{ccc}
1 & -b & -c+a b \\
0 & 1 & -a \\
0 & 0 & 1
\end{array}\right)
$$

and

$$
\sigma\left(\begin{array}{ccc}
1 & a & c \\
0 & 1 & b \\
0 & 0 & 1
\end{array}\right)=\left(\begin{array}{ccc}
1 & -a & -c+a b \\
0 & 1 & -b \\
0 & 0 & 1
\end{array}\right)
$$


It easy to see that

$$
\sigma \circ \tau=\tau \circ \sigma\left(\begin{array}{lll}
1 & a & c \\
0 & 1 & b \\
0 & 0 & 1
\end{array}\right)=\left(\begin{array}{ccc}
1 & b & c \\
0 & 1 & a \\
0 & 0 & 1
\end{array}\right) .
$$

The continuous non-zero multiplicative functions on $G$ are given (see [ [24]):

$$
\mu_{\alpha, \beta}(a, b)=\exp ^{\alpha a+\beta b}, \quad \alpha, \beta \in \mathbb{C} .
$$

We compute that $\mu=\mu \circ \sigma$ if and only if $\beta=-\alpha$, and that $\mu=\mu \circ \tau$ if and only if $\alpha=\beta=0$. Then

$$
f((a, b))=\left(\exp ^{\alpha(a-b)}+\exp ^{\alpha(b-a)}\right) / 2
$$

By [24, Theorem 8.29.] Any continuous solution of the pre-d'Alembert functional equation on $G$, is abelian. In particular it has no non-abelian solution on $G$.

\section{REFERENCES}

[1] J. Aczél and J. Dhombres: Functional equations in several variables with applications to mathematics, information theory and to the natural and social sciences. Encyclopedia of Mathematics and its Applications, vol. 31. Cambridge University Press, Cambridge, (1989).

[2] R. Badora, On a joint generalization of Cauchy's and d'Alembert's functional equations. Aequationes Math. 43 (1992), 72-89.

[3] B. Bouikhalene, E. Elqorachi, An extension of Van Vleck's functional equation for the sine, Acta Math. Hungar. 150 (2016), no. 1, 258-267.

[4] W. Chojnacki, On some functional equation generalizing Cauchy's and d'Alembert's functional equations. Colloq. Math. 55 (1988), 169-178.

[5] T.M.K. Davison, D'Alembert's functional equation on topological groups. Aequationes Math. 76 (2008), 33-53.

[6] T.M.K. Davison, D'Alembert's functional equation on topological monoids. Publ. Math. Debrecen. 75 (2009), 41-66.

[7] B.R. Ebanks, Some trigonometric functional equations on monoids generated by their squares. Aequationes. Math. (2020), https://doi.org/10.1007/s00010-020-00730-5.

[8] B.R. Ebanks and H. Stetkær, On Wilson's functional equations. Aequationes math. 89, (2015), 339-354.

[9] B.R. Ebanks and H. Stetkær, d'Alembert's other functional equation on monoids with an involution. Aequationes Math. 89 (2015), 187-206.

[10] E. Elqorachi, A. Redouani, Solutions and stability of a variant of Wilson's functional equation. Proyecciones. 37 (2018), no. 2, 317-344.

[11] Iz. El-fassi, A. Chahbi, S. Kabbaj, The Solution of a Class Functional Equations on Semi-Groups. Filomat. 31:14 (2017), 4613-4618.

[12] E. Elqorachi, Integral Van Vleck's and Kannappan's functional equations on semigroups. Aequationes Math. 91 (2017), no. 1, 83-98.

[13] E. Elqorachi, A. Redouani , Th.M. Rassias, Solutions and stability of a variant of Van Vleck's and d'Alembert's functional equations. Int. J. Nonlinear Anal. Appl. 7 (2016), no. 2, 279-301.

[14] B. Fadli, D. Zeglami, S. Kabbaj, A variant of Wilson's functional equation. Publ. Math. Debrecen. 87/3-4 (2015), 415-427.

[15] Che Tat Ng, Hou Yu Zhao, and Xia Lin, A functional equation on groups with involutions. Aequationes. Math. (2019) https://doi.org/10.1007/s00010-019-00688-z.

[16] V. Lomonosov, P. Rosenthal, The simplest proof of Burnside's theorem on matrix algebra. Linear Algebra Appli. 383 (2004), 45-47.

[17] A. M. Perkins, P. K. Sahoo, On two functional equations with involution on groups related to sine and cosine functions. Aequationes Math. 89 (2015), 1251-1263.

[18] PI. Kannappan, A functional equation for the cosine. Canad. Math. Bull. 2 (1968), 495-498. 
[19] K.H. Sabour, B. Fadli, S. Kabbaj, Wilson's functional eqaution on monoids with involutive automorphisms. Aequationes Math. 90, (2016), 1001-1011.

[20] H. Stetkær, The small dimension lemma and d'Alembert's equation on semigroups. Aequationes Math. (2020), https://doi.org/10.1007/s00010-020-00746-x

[21] Stetkær H., Kannappan's functional equation on semigroups with involution. Semigroup Forum. 94 (2017), 17-33.

[22] H. Stetkær, Van Vleck's functional equation for the sine. Aequationes Math. 90 (2016), no. 1, 25-34.

[23] H. Stetkær, A variant of d'Alemberts functional equation, Aequationes Math. 89 (2015), 657-662.

[24] H. Stetkær, Functional Equations on Groups. World Scientfic, Singapore, (2013).

[25] H. Stetkær, Properties of d'Alembert functions. Aequationes Math. 77 (2009), 281-301.

[26] H. Stetkær, On a variant of Wilson's functional equation on groups. Aequationes. Math. 68(3), (2004) 160-176.

[27] H. Stetkær, D'Alembert's and Wilson's functional equations on step 2 nilpotent groups. Aequationes Math. 67 (2004), 241-262.

[28] H. Stetkær, D'Alembert's functional equations on metabelian groups. Aequationes Math. 59 (2000), 306-320.

[29] E.B. Van Vleck, A functional equation for the sine. Ann. of Math. (2) 11 (1910), no. 4, 161-165.

[30] E.B. Van Vleck, On the functional equation for the sine. Additional note on: A functional equation for the sine. Ann. of Math. (2) 13 (1911/12), no. 1-4, 154.

Ibn Zohr University, Faculty of Sciences, Department of Mathematics, Agadir, Morocco

Email address: abdellatifchahbi@gmail.com-elqorachi@hotmail.com 